\title{
On stability and stabilization of singular uncertain Takagi-Sugeno fuzzy systems ${ }^{\text {th }}$
}

\author{
M. Chadli ${ }^{\mathrm{a}, *}$, H.R. Karimi ${ }^{\mathrm{b}}$, P. Shi ${ }^{\mathrm{c}, \mathrm{d}}$ \\ anniversity of Picardie Jules Verne, Laboratory of "Modélisation, Information et Systèmes" (MIS), \\ 33 rue Saint-Leu, 80039 Amiens, France \\ ${ }^{\mathrm{b}}$ Department of Engineering, Faculty of Engineering and Science, University of Agder, 4898 Grimstad, Norway \\ ${ }^{\mathrm{c}}$ College of Engineering and Science, Victoria University, Melbourne, VIC 8001, Australia \\ ${ }^{\mathrm{d}}$ School of Electrical and Electronic Engineering, The University of Adelaide, Adelaide, SA 5005, Australia
}

Received 12 July 2006; received in revised form 7 October 2013; accepted 11 November 2013

Available online 28 November 2013

\begin{abstract}
This paper deals with the problem of robust stability and robust stabilization for a class of continuous-time singular Takagi-Sugeno fuzzy systems. Sufficient conditions on stability and stabilization are proposed in terms of strict LMI (Linear Matrix Inequality) for uncertain T-S fuzzy models. In order to reduce the conservatism of results developed using quadratic method, an approach based on non-quadratic Lyapunov functions and S-procedure is proposed. Illustrative examples are given to show the effectiveness of the given results
\end{abstract}

\section{Introduction}

There have been several studies on analysis and design problems for Takagi-Sugeno models [1] based on Lyapunov theory and LMI (Linear Matrix Inequality) formulations [2]. These studies use quadratic Lyapunov functions to derive sufficient conditions for the stability and stabilization (see among others [6-8]) and non-quadratic Lyapunov functions [4,5,9-13] for less conservatism. In the last few decades, there have been considerable research efforts on the study of singular systems. This is due to the extensive applications of singular systems in many practical systems,

\footnotetext{
* *orresponding author. Tel.: +33 322825918.

E-mail addresses: mchadli@u-picardie.fr, mohammed.chadli@u-picardie.fr (M. Chadli).
} 
such as circuits, boundary control systems, chemical processes, and other areas. For more details on this, we refer the reader to [14-17] and the references therein. Singular systems are also referred to as descriptor systems, implicit systems, generalized state-space systems, differentialalgebraic systems or semi-state systems [14,15]. A great number of fundamental notions and results in control and systems theory based on state-space systems have been successfully extended to singular systems (see [18-28] and the references therein). Recently stability and stabilization of singular fuzzy systems [29-39] and singular linear systems have been addressed (see [40-42] and reference therein). However, up to date and to the best of our knowledge, the class of singular fuzzy systems has not yet been fully investigated and this will be the goal of this paper.

Most of the results developed for this class of systems are not easily tractable and often conservative. All these results are established using a quadratic Lyapunov function with common symmetric and positive-definite matrix that leads to some conservatism. In order to relax these previous derived constraints, our goal is to extend results on non-quadratic stability to the class of singular fuzzy systems. Both problems of robust stability and robust stabilization for a class of continuous-time singular fuzzy models with norm-bounded uncertainties are considered in LMI formulation. Finally, a numerical example is provided to demonstrate the effectiveness of the proposed methods.

The rest of this paper is organized as follows. In Section 2, the problem is formulated and the goal of the paper is stated. In Section 3, the main results are given and these include results on stability and robust stability.

Notation: The notation $X \geq Y$ (respectively, $X>Y$ ), where $X$ and $Y$ are symmetric matrices, means that $X-Y$ is positive semi-definite (respectively, positive definite), $\mathbb{R}^{n}$ and $\mathbb{R}^{n \times m}$ denote, respectively, the $n$ dimensional Euclidean space and the set of all $n \times m$ real matrices. The superscript "T" denotes matrix transposition, symbol (*) denotes the transpose elements in the symmetric position and $\llbracket$ is the identity matrices with compatible dimensions and $\mathcal{I}=\{1,2, \ldots, r\}$. For a matrix $Z \in \mathbb{R}^{n \times m}$ with rank $Z=n_{z}<n$, let $Z^{\perp} \in \mathbb{R}^{n \times\left(n-n_{z}\right)}$ be any matrix such that $Z Z^{\perp}=0$ and $Z^{\perp \top} Z^{\perp}>0$. When $Z$ is of full column rank matrix, we take $Z^{\perp}=0$ by convention.

\section{Problem statement}

T-S models are described by fuzzy rules of the type IF-THEN that represent local input output models for nonlinear systems. The considered singular fuzzy systems with $r$ - $\left(M_{i 1}, \ldots, M_{i r}\right)$ fuzzy sets are described as follows:

Plant rule $i$ : IF $z_{1}(t) \in M_{i 1}$ and $\cdots$ and $z_{r}(t) \in M_{i r}$

$$
\left\{\begin{array}{l}
E \dot{x}(t)=\widehat{A}_{i} x(t)+\widehat{B}_{i} u(t), \quad i \in \mathcal{I} \\
y(t)=C x(t)
\end{array}\right.
$$

with $\widehat{A}_{i}=A_{i}+\Delta A_{i}(t)$ and $\widehat{B}_{i}=B_{i}+\Delta B_{i}(t)$. Then, the overall singular fuzzy system is described by

$$
\left\{\begin{array}{l}
E \dot{x}(t)=\sum_{i=1}^{r} \mu_{i}(z(t))\left(\widehat{A}_{i} x(t)+\widehat{B}_{i} u(t)\right) \\
y(t)=C x(t)
\end{array}\right.
$$


where

$$
\mu_{i}(z(t))=\frac{w_{i}(z(t))}{\sum_{i=1}^{r} w_{i}(z(t))} \geq 0, \quad \sum_{i=1}^{r} \mu_{i}(z(t))=1
$$

with $w_{i}(z(t))=\prod_{j=1}^{r} M_{i j}\left(z_{j}(t)\right), r$ is the number of sub-models and $z(t)$ is the premise variable depending on measurable variables. $x(t) \in \mathbb{R}^{n}$ is the state vector, $A_{i} \in \mathbb{R}^{n \times n}$ are the $i$ th state matrix, $\Delta A_{i}(t)$ and $\Delta B_{i}(t)$ are time-varying matrices representing parametric uncertainties in the plant model. The matrix $E$ may be singular with $0 \leq \operatorname{rank}(E)=n_{E}<n$. When the matrix $E$ is nonsingular, the system (2) is referred to as regular system.

The considered uncertainties in this note are known in the literature as norm-bounded uncertainties, they are admissibly norm-bounded, structured and satisfy

$$
\Delta A_{i}(t)=D_{A_{i}} F_{A_{i}}(t) E_{A_{i}}, \quad \Delta B_{i}(t)=D_{B_{i}} F_{B_{i}}(t) E_{B_{i}}
$$

with $D_{*_{i}}$ and $E_{*_{i}}$ are known real matrices with appropriate dimensions and $F_{*_{i}}$ satisfies

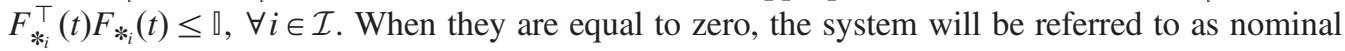
system.

Now consider the nominal part of descriptor fuzzy system (2) with $u(t)=0$ in the following:

$$
E \dot{x}(t)=\sum_{i=1}^{r} \mu_{i}(z(t)) A_{i} x(t)
$$

The descriptor model (4) is said to be stable if $d V(x(t)) / d t \leq-\alpha\|x(t)\|_{2}, \alpha>0$ for a given Lyapunov function candidate $V(x(t)) \geq 0, \forall x(t) \neq 0$ and the following conditions are satisfied [14]:

(i) $\operatorname{det}\left(s E-A_{i}\right)$ is not identically zero, i.e. nominal system (4) is said to be locally regular. (ii) $\operatorname{deg}\left(\operatorname{det}\left(s E-A_{i}\right)\right)=\operatorname{rank}(E)$, i.e. nominal system (4) is said to be locally impulse free.

In the rest of the paper, we will assume that the solution of the system (4) exits. In this paper we are interested in developing sufficient conditions that can be used to check if the given system in Eq. (4) is stable and robust stable in case of the presence of norm-bounded uncertainties. Our methodology in this paper will be mainly based on the Lyapunov theory and LMI formulation.

The following useful lemmas [2,3] will be using in the rest of the paper.

Lemma 1. Let $H$ and $E$ be given matrices with appropriate dimensions and $F$ satisfying

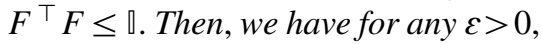

$$
H F E+E^{\top} F^{\top} H^{\top} \leq \varepsilon H H^{\top}+\frac{1}{\varepsilon} E^{\top} E .
$$

Lemma 2. Let $F_{0}(x(t)), \ldots, F_{q}(x(t))$ be quadratic functions of the variable $x(t) \in \mathbb{R}^{n}$. If there exist positive scalars $\tau_{1}, \ldots, \tau_{q}$ such that $F_{0}(x(t))-\sum_{i=1}^{q} \tau_{i} F_{i}(x(t)) \leq 0$, then $F_{0}(x(t)) \leq 0$ for all $x(t)$ such that $F_{i}(x(t)) \leq 0$, for $i \in\{1,2, \ldots, q\}$.

Lemma 3. Condition $\sum_{i=1}^{r} \sum_{j=1}^{r} \mu_{i}(z(t)) \mu_{j}(z(t)) \Gamma_{i j}<0$ is fulfilled provided the following conditions hold $\forall(i, j) \in \mathcal{I}^{2}$ and $i \neq j$ :

$$
\begin{aligned}
& \Gamma_{i i}<0 \\
& \frac{2}{r-1} \Gamma_{i i}+\Gamma_{i j}+\Gamma_{j i} \leq 0
\end{aligned}
$$




\section{Main results}

In this section, result assuring the global asymptotic stability of system (4) is proposed in terms of non-strict LMIs. Based on this result, strict LMI conditions are developed. Then, these results are extended to the case of uncertain $\mathrm{T}-\mathrm{S}$ descriptor systems.

\subsection{Stability analysis}

The following theorem gives global asymptotically stable of nominal system (4).

Theorem 1. The singular fuzzy system (4) is stable if there exist nonsingular matrices $P_{i}$ and positive scalars $\tau_{i j k}$ such that the following matrix inequalities hold for each $(i, k) \in \mathcal{I}^{2}$ :

$$
\begin{aligned}
& E^{\top} P_{i}=P_{i}^{\top} E \geq 0 \\
& A_{i}^{\top} P_{k}+P_{k}^{\top} A_{i}+\sum_{j=1}^{r} \tau_{i j k} E^{\top}\left[P_{k}-P_{j}\right]<0
\end{aligned}
$$

Proof. Let us choose the following Lyapunov function:

$$
V_{k}(x(t))=x^{\top}(t) E^{\top} P_{k} x(t), \quad E^{\top} P_{k}=P_{k}^{\top} E \geq 0, k \in \mathcal{I}
$$

and define the Lyapunov function candidate as follows:

$$
V(x(t))=\max \left\{V_{i}(x(t))\right\}=\max \left\{V_{1}(x(t)), V_{2}(x(t)), \ldots, V_{r}(x(t))\right\},
$$

The derivative of $V(x)$ with respect to time $t$ along the system trajectory (2) is given for all $k \in \mathcal{I}$ by

$$
\dot{V}(x)=\dot{V}_{k}(x(t)) \quad \text { if } E^{\top} P_{k} \geq E^{\top} P_{j} \text {, for all } j \in \mathcal{I}
$$

Therefore, when $E^{\top} P_{k} \geq E^{\top} P_{j}$, for all $j \in \mathcal{I}$, we have

$$
\dot{V}(x)=\dot{x}^{\top}(t) E^{\top} P_{k} x(t)+x^{\top}(t) E^{\top} P_{k} \dot{x}(t)=x^{\top}(t) \sum_{i=1}^{r} \mu_{i}(z(t))\left[A_{i}^{\top} P_{k}+P_{k}^{\top} A_{i}\right] x(t)
$$

Consequently if for all $j \in \mathcal{I}$ we have $A_{i}^{\top} P_{k}+P_{k}^{\top} A_{i}<0$ when $E^{\top} P_{k} \geq E^{\top} P_{j}$, we get $\dot{V}(x)<0$. Using Lemma 2, the conditions (8) (9) are obtained.

Corollary 1. The nominal singular fuzzy system of $E q$. (2) with $u(t)=0$ is stable if there exist nonsingular matrices $P_{i}$ and positive scalars $\tau_{i j k}$ such that the following matrix inequalities hold for each $(i, k) \in \mathcal{I}^{2}$ :

$$
\begin{aligned}
& E P_{i}=P_{i}^{\top} E^{\top} \geq 0 \\
& A_{i} P_{k}+P_{k}^{\top} A_{i}^{\top}+\sum_{j=1}^{r} \tau_{i j k} E\left[P_{k}-P_{j}\right]<0
\end{aligned}
$$

Proof. The proof is similar to the one of Theorem 1 by considering the dual system $E^{\top} \dot{x}(t)=\sum_{i=1}^{r} \mu_{i}(z(t)) A_{i}^{\top} x(t)$.

Remark 1. The conditions of Theorem 1 include the quadratic conditions. In fact when $P_{i}=P$, $\forall i \in \mathcal{I}$, we have $V(x(t))=\max \left\{V_{1}(x(t)), V_{2}(x(t)), \ldots, V_{r}(x(t))\right\}=x^{\top}(t) E^{\top} P x(t)$ and $E^{\top} P=$ $P^{\top} E \geq 0$. 
The proposed result of Theorem 1 gives sufficient stability conditions in terms of non-strict LMIs which contain equality constraints [43]. This may lead to numerical problems since equality constraints are usually not satisfied perfectly. Thus, from the numerical point of view, strict LMI conditions are more suitable than non-strict ones. In order to avoid this problem, the following result gives strict LMI conditions.

Theorem 2. The nominal singular fuzzy system of $E q$. (2) with $u(t)=0$ is stable if there exist matrices $X_{i}>0$ and $Q_{i}$ and positive scalars $\tau_{i j k}$ such that the following matrix inequalities hold for each $(i, k) \in \mathcal{I}^{2}$ :

$$
A_{i}^{\top}\left(X_{k} E+Z Q_{k}\right)+\left(X_{k} E+Z Q_{k}\right)^{\top} A_{i}+\sum_{j=1}^{r} \tau_{i j k} E^{\top}\left[X_{k}-X_{j}\right] E<0
$$

with $E^{\top} Z=0$ and $Z \in \mathbb{R}^{n \times\left(n-n_{E}\right)}$.

Proof. Let $P_{i}=X_{i} E+Z Q_{i}$. Then, it is easy to see that $E^{\top} P_{i}=P_{i}^{\top} E=E^{\top} X_{i} E \geq 0$ and $E^{\top}\left[P_{k}-P_{j}\right]=E^{\top}\left[X_{k}-X_{j}\right] E$. Consequently, conditions of Theorem 2 lead to the one of Theorem 1 .

With the same reasoning, the following dual form of Theorem 2 could be proved:

$$
A_{i}\left(X_{k} E^{\top}+Z Q_{k}\right)+\left(X_{k} E^{\top}+Z Q_{k}\right)^{\top} A_{i}^{\top}+\sum_{j=1}^{r} \tau_{i j k} E\left[X_{k}-X_{j}\right] E^{\top}<0
$$

with $E Z=0$.

The conditions of Theorem 1 are bilinear in the variables $P_{i}, \forall i \in \mathcal{I}$, and $\tau_{i j k}$ for all $(i, j, k) \in \mathcal{I}^{3}$. The solution in some cases cannot easily be found. To overcome this conservatism another way to keep this relaxation, with transformation of the conditions of Theorem 1 in LMI formulation in $P_{i}, i \in \mathcal{I}$, consists of fixing the real parameters $\tau_{i j k}$, and solving the resulting coupled set of LMIs.

Now consider uncertain singular fuzzy models (2). Based on the results of Theorem 1, the following theorem proposes robust stability conditions in strict LMI terms.

Theorem 3. The uncertain singular fuzzy system (2) with $u(t)=0$ is stable if there exist matrices $X_{i}>0$ and $Q_{i}$, positive real numbers $\varepsilon_{A_{i}}$ and $\tau_{i j k}$ such that the following matrix inequalities hold for all $(i, k) \in \mathcal{I}^{2}$ :

$$
\left[\begin{array}{cc}
\Omega_{i k} & \left(X_{k} E+Z Q_{k}\right)^{\top} D_{A_{i}} \\
(*) & -\varepsilon_{i} \rrbracket
\end{array}\right]<0
$$

with $E^{\top} Z=0, Z \in \mathbb{R}^{n \times\left(n-n_{E}\right)}$ and

$$
\Omega_{i k}=\left(X_{k} E+Z Q_{k}\right)^{\top} A_{i}+A_{i}^{\top}\left(X_{k} E+Z Q_{k}\right)+\varepsilon_{i} E_{A_{i}}^{\top} E_{A_{i}}+\sum_{j=1}^{r} \tau_{i j k} E^{\top}\left[X_{k}-X_{j}\right] E
$$

Proof. The dynamics of the uncertain singular fuzzy models (2) is stable if the conditions of Theorem 2 hold where $A_{i}$ is substituted by $A_{i}+\Delta A_{i}(t)$, i.e.:

$$
P_{k}^{\top} A_{i}+A_{i}^{\top} P_{k}+P_{k}^{\top} D_{A_{i}} F_{A_{i}} E_{A_{i}}+E_{A_{i}}^{\top} F_{A_{i}}^{\top} D_{A_{i}}^{\top} P_{k}+\sum_{j=1}^{r} \tau_{i j k} E^{\top}\left[X_{k}-X_{j}\right] E<0
$$


Using Lemma 1, we have for any $\varepsilon_{i}>0, i \in \mathcal{I}$ :

$P_{k}^{\top} D_{A_{i}} F_{A_{i}} E_{A_{i}}+E_{A_{i}}^{\top} F_{A_{i}}^{\top} D_{A_{i}}^{\top} P_{k} \leq \varepsilon_{i}^{-1} P_{k}^{\top} D_{A_{i}} D_{A_{i}}^{\top} P_{k}+\varepsilon_{i} E_{A_{i}}^{\top} E_{A_{i}}(20)$ Applying the Schur complement on this inequality, the results for the robust stability is

obtained.

Remark 2. The same procedures used to solve the conditions of Theorem 2 can be used to transform the conditions of Theorem 2 to a tractable problem that can be solved using classical numerical tools.

\subsection{Robust stabilization}

In this section robust stabilization of closed-loop model (2) is studied by considering the control law

$$
u(t)=\sum_{i=1}^{r} \mu_{i}(z(t)) K_{i} x(t)
$$

where $K_{i}$ are the gain parameters to be determined. Then, closed-loop model (2) becomes

$$
E \dot{x}(t)=\sum_{i=1}^{r} \sum_{l=1}^{r} \mu_{i}(z(t)) \mu_{l}(z(t)) \widehat{\mathbf{A}}_{i l} x(t)
$$

with $\widehat{\mathbf{A}}_{i l}=\widehat{A}_{i}+\widehat{B}_{i} K_{l}$.

Note that a robust control law could be proposed by using directly the above results, i.e. Theorem 2 for the nominal case and Theorem 3 for the uncertain one. However the obtained conditions lead to nonlinear constraints very difficult to solve with existing numerical tools. In the following, sufficient stability conditions to design control law (21) are given by decoupling the product of Lyapunov matrices by $K_{i}$ and introducing free variables $G$.

Theorem 4. The uncertain singular fuzzy system (22) is stable if there exist matrices $X_{i}>0$, $G$ and $Q$, positive real numbers $\varepsilon_{i}$ and $\tau_{i j k}$ such that the following matrix inequalities hold for all $(i, k) \in \mathcal{I}^{2}$ and $i \neq k$ :

$$
\Sigma_{i k}<0
$$

$$
\frac{2}{r-1} \Sigma_{i i}+\Sigma_{i k}+\Sigma_{k i} \leq 0
$$

with $E Z=0, \quad \psi_{i k}=\sum_{j=1}^{r} \tau_{i j k} E\left[X_{k}-X_{j}\right] E^{\top}+\phi_{i k}+\phi_{i k}^{\top}+\varepsilon_{i k}\left(D_{A_{i}} D_{A_{i}}^{\top}+D_{B_{i}} D_{B_{i}}^{\top}\right), \quad \phi_{i k}=A_{i} G+$ $B_{i} Y_{k}$ and

$$
\Sigma_{i k}=\left[\begin{array}{cccc}
\psi_{i k} & (*) & (*) & (*) \\
\left(X_{k} E^{\top}+Z Q_{k}\right)+\phi_{i k}^{\top}-G & -G-G^{\top} & (*) & (*) \\
E_{A_{i}} G & E_{A_{i}} G & -\varepsilon_{i k} \square & 0 \\
E_{B_{i}} Y_{k} & E_{B_{i}} Y_{k} & 0 & -\varepsilon_{i k} \square
\end{array}\right]
$$

The control gains are calculated by

$$
K_{i}=Y_{i} G^{-1}
$$


Proof. Based on the dual condition of Theorem 2, the dynamics of the uncertain singular fuzzy models (22) is stable if the following conditions hold:

$$
\widehat{\mathbf{A}}_{i l}\left(X_{k} E^{\top}+Z Q_{k}\right)+\left(X_{k} E^{\top}+Z Q_{k}\right)^{\top} \widehat{\mathbf{A}}_{i l}^{\top}+\sum_{j=1}^{r} \tau_{i j k} E\left[X_{k}-X_{j}\right] E^{\top}<0
$$

In order to decouple the product of Lyapunov matrices $X_{i}$ by $K_{i}$ and introducing free variables $G$, the following sufficient conditions are proposed:

$$
\left[\begin{array}{cc}
\sum_{j=1}^{r} \tau_{i j k} E\left[X_{k}-X_{j}\right] E^{\top}+\widehat{\mathbf{A}}_{i l} G+G^{\top} \widehat{\mathbf{A}}_{i l}^{\top} & \left(X_{k} E^{\top}+Z Q_{k}\right)^{\top}+\widehat{\mathbf{A}}_{i l} G-G^{\top} \\
* & -G-G^{\top}
\end{array}\right]<0
$$

Note that to get Eq. (27), it suffices to pre-multiply Eq. (28) by $\left[I \widehat{\mathbf{A}}_{i l}\right]$ and post-multiplying it by $\left[I \widehat{\mathbf{A}}_{i l}\right]^{\top}$ where $G$ is a free nonsingular matrix. Knowing that $\widehat{\mathbf{A}}_{i l} G=\phi_{i j}+\Delta A_{i}(t) G+\Delta B_{i}(t) Y_{l}$ with $\phi_{i l}=A_{i} G+B_{i} Y_{l}$ and $Y_{i}=K_{i G}$, conditions (28) can be rewritten with $l=k$ as follows:

$$
\begin{aligned}
\Omega_{i k}= & {\left[\begin{array}{cc}
\sum_{j=1}^{r} \tau_{i j k} E\left[X_{k}-X_{j}\right] E^{\top}+\phi_{i k}+\phi_{i k}^{\top} & \left(X_{k} E^{\top}+Z Q_{k}\right)^{\top}+\phi_{i k}-G^{\top} \\
(*) & -G-G^{\top}
\end{array}\right] } \\
& +\left[\begin{array}{cc}
\Delta A_{i}(t) G+\Delta B_{i}(t) Y_{k}+\left(\Delta A_{i}(t) G+\Delta B_{i}(t) Y_{k}\right)^{\top} & \Delta A_{i}(t) G+\Delta B_{i}(t) Y_{k} \\
(*) & 0
\end{array}\right]
\end{aligned}
$$

The second term on the right-hand side of Eq. (29) can be written as

$$
\begin{gathered}
{\left[\begin{array}{cc}
\Delta A_{i}(t) G+\Delta B_{i}(t) Y_{k}+\left(\Delta A_{i}(t) G+\Delta B_{i}(t) Y_{k}\right)^{\top} & \Delta A_{i}(t) G+\Delta B_{i}(t) Y_{k} \\
(*) & 0
\end{array}\right]} \\
=\left[\begin{array}{cc}
D_{A_{i}} & D_{B_{i}} \\
0 & 0
\end{array}\right]\left[\begin{array}{cc}
F_{A_{i}}(t) & 0 \\
0 & F_{B_{i}}(t)
\end{array}\right]\left[\begin{array}{cc}
E_{A_{i}} G & E_{A_{i}} G \\
E_{B_{i}} Y_{k} & E_{B_{i}} Y_{k}
\end{array}\right]+(*)
\end{gathered}
$$

Applying Lemma 1 and the Schur complement to Eq. (29) with Eq. (30), we get $\Sigma_{i k}<0$ where $\Sigma_{i k}$ is defined in Eq. (25). Using Lemma 3, we obtain conditions (23) and (24). This completes the proof.

Remark 3. This formulation has the advantage to remove the equality constraint involving singular matrix $E$ and the control gains are dependent on supplementary free variable $G$ instead of Lyapunov matrices. These strict LMIs design conditions can be solved using existing numerical tools. This formulation covers existing results and leads to less of conservatism for the design problems. 


\section{Numerical examples}

\subsection{Robust stability analysis}

To show the validness of our result, let us consider a numerical example of two uncertain submodels as follows:

$$
\begin{aligned}
& A_{1}=\left[\begin{array}{ccc}
-1.0 & -0.1 & -0.2 \\
0.0 & 0.0 & 1.0 \\
0.0 & -0.06 & -1.0
\end{array}\right], \quad A_{2}=\left[\begin{array}{ccc}
-1.0 & -0.1 & 0.0 \\
0.0 & -0.5 & 1.0 \\
0.0 & -1.94 & -1.0
\end{array}\right] \\
& D_{A_{1}}=D_{A_{2}}=\left[\begin{array}{l}
0.01 \\
0.02 \\
0.02
\end{array}\right], \quad E_{A_{1}}=E_{A_{2}}=\left[\begin{array}{l}
0.03 \\
0.02 \\
0.02
\end{array}\right]^{T}
\end{aligned}
$$

The singular matrix, $E$, is given by

$$
E=\left[\begin{array}{ccc}
1 & 1 & 0 \\
0.1 & 1 & 1 \\
0 & 0 & 0
\end{array}\right]
$$

Solving the conditions (17), we get the following feasible problem:

$$
P_{1}=\left[\begin{array}{ccc}
1.2080 & 0.4847 & 1.1553 \\
-0.7060 & 0.1723 & -0.3908 \\
1.1162 & 0.7645 & 1.5245
\end{array}\right], \quad P_{2}=\left[\begin{array}{ccc}
1.8741 & 0.5974 & 1.3532 \\
-1.2871 & -0.1044 & -0.8313 \\
1.2700 & 0.5218 & 1.3634
\end{array}\right]
$$

with $\varepsilon_{A_{1}}=1.7329, \varepsilon_{A_{2}}=0.5966$, and $\tau_{112}=0, \tau_{121}=0.1, \tau_{221}=0.7$ and $\tau_{212}=0$.

Consequently, we can conclude that the system is robust stable. However, quadratic conditions given in [30] are not feasible and then fail to prove the stability of this example. This illustrative example shows that the given stability conditions introduce more of relaxation and allow to improve the results obtained by the quadratic method with or without uncertainties.

\subsection{Robust stabilization}

Now, consider the following uncertain T-S model with four sub-models as follows:

$$
\begin{aligned}
& A_{1}=\left[\begin{array}{ccc}
0.20 & 0 & 10.00 \\
-4.00 & -7.00 & 0 \\
1.0 & 0 & 0.10
\end{array}\right], A_{2}=\left[\begin{array}{ccc}
0.20 & 0 & 13.00 \\
-4.00 & -10.00 & 0 \\
1.00 & 0 & 0
\end{array}\right] \\
& A_{3}=\left[\begin{array}{ccc}
0.50 & 0 & 13.00 \\
-1.00 & -13.00 & 0 \\
1.00 & 0 & 0.30
\end{array}\right], A_{4}=\left[\begin{array}{ccc}
0.50 & 0 & 10.00 \\
-1.00 & -10.00 & 0 \\
1.00 & 0 & 0
\end{array}\right]
\end{aligned}
$$




$$
\begin{aligned}
& B_{1}=\left[\begin{array}{c}
0.2 \\
1.0 \\
-1.0
\end{array}\right], \quad B_{2}=\left[\begin{array}{c}
0.5 \\
1.0 \\
-1.2
\end{array}\right], \quad B_{3}=\left[\begin{array}{c}
0.3 \\
1.0 \\
-1.0
\end{array}\right], \quad B_{4}=\left[\begin{array}{c}
0.2 \\
1.0 \\
-1.2
\end{array}\right] \\
& D_{A_{1}}=\left[\begin{array}{l}
0.1 \\
0.2 \\
0.3
\end{array}\right], \quad D_{A_{2}}=\left[\begin{array}{c}
0.1 \\
-0.1 \\
-0.5
\end{array}\right], \quad D_{A_{3}}=\left[\begin{array}{l}
0.1 \\
0.4 \\
0.2
\end{array}\right], \quad D_{A_{4}}=\left[\begin{array}{c}
0.1 \\
-0.5 \\
-0.1
\end{array}\right] \\
& E_{A_{1}}^{T}=\left[\begin{array}{c}
-0.5 \\
-0.5 \\
-0.5
\end{array}\right], \quad E_{A_{2}}^{T}=\left[\begin{array}{l}
0.2 \\
0.2 \\
0.5
\end{array}\right], \quad E_{A_{3}}^{T}=\left[\begin{array}{l}
0.5 \\
0.5 \\
0.5
\end{array}\right], \quad E_{A_{4}}^{T}=\left[\begin{array}{c}
-0.21 \\
-0.3 \\
0.5
\end{array}\right] \\
& D_{B_{1}}^{T}=\left[\begin{array}{c}
-0.2 \\
-1.5 \\
0.5
\end{array}\right]
\end{aligned}
$$

with $D_{B_{1}}=D_{B_{2}}=D_{B_{3}}=D_{B_{4}}, E_{B_{1}}=0.5, E_{B_{2}}=1, E_{B_{3}}=1$ and $E_{B_{1}}=0.5$. The singular matrix is

$$
E=\left[\begin{array}{lll}
1.2 & 0 & 0.0 \\
0.0 & 1 & 0.5 \\
0.0 & 0 & 0.0
\end{array}\right]
$$

Then $Z^{T}=[0,-0.5,1.0]$. The resolution of the conditions of Theorem 4 leads to feasible problem:

$$
\begin{aligned}
& X_{1}=10^{3}\left[\begin{array}{ccc}
0.7178 & 0.3942 & 0.1971 \\
0.3942 & 4.1415 & -0.5731 \\
0.1971 & -0.5731 & 5.0012
\end{array}\right], \quad X_{2}=10^{3}\left[\begin{array}{cccc}
0.0290 & 0.2708 & 0.1354 \\
0.2708 & 5.5791 & 0.1457 \\
0.1354 & 0.1457 & 5.3606
\end{array}\right] \\
& X_{3}=10^{3}\left[\begin{array}{lll}
0.2047 & 0.1679 & 0.0839 \\
0.1679 & 6.2159 & 0.4641 \\
0.0839 & 0.4641 & 5.5198
\end{array}\right], \quad X_{4}=10^{3}\left[\begin{array}{ccc}
3.4417 & -0.4934 & -0.2467 \\
-0.4934 & 5.8729 & 0.2925 \\
-0.2467 & 0.2925 & 5.4340
\end{array}\right] \\
& G=10^{3}\left[\begin{array}{ccc}
1.2899 & 0.0661 & -0.4220 \\
-0.1798 & 0.5444 & 0.0464 \\
-0.2601 & -0.0689 & 0.1121
\end{array}\right]
\end{aligned}
$$

We get the controller gains:

$$
\begin{aligned}
& K_{1}=[3.9406,1.1107,14.5782], \quad K_{2}=[1.7050,0.3506,5.9574] \\
& K_{3}=[2.3766,0.4073,8.7317], \quad K_{4}=[4.4962,0.7605,16.4140]
\end{aligned}
$$

As stated above, quadratic conditions derived in [30] fail to prove the stability of the given example with or without uncertainties. This example shows that the derived stability conditions allow to improve the results obtained by the quadratic method. 


\section{Conclusion}

This note deals with the problems of robust stability and stabilization for a class of continuoustime singular Takagi-Sugeno fuzzy systems. Strict LMI conditions are given for stability, stabilization and their robustness. The derived stability conditions are proved to be less conservative than previous results and particularly include quadratic conditions. The LMI framework is used to establish the different results on stability and its robustness. The developed results can easily be solved using existing numerical tools. Examples, where quadratic conditions fail to show the stability, are given to illustrate the advantage of the proposed results.

\section{References}

[1] T. Takagi, M. Sugeno, Fuzzy identification of systems and its application to modelling and control, IEEE Trans. Syst. Man Cybern. 15 (1) (1985) 116-132.

[2] S. Boyd, et al., Linear Matrix Inequalities in Systems and Control Theory, SIAM, Philadelphia, PA, 1994.

[3] H.D. Tuan, P. Apkarian, T. Narikiyo, Y. Yamamoto, Parameterized linear matrix inequality techniques in fuzzy control system design, IEEE Trans. Fuzzy Syst. 9 (2) (2001) 324-332.

[4] A. Sala, C. Ario, Asymptotically necessary and sufficient conditions for stability and performance in fuzzy control: applications of Polya's theorem, Fuzzy Sets Syst. 158 (2007) 2671-2686.

[5] A. Sala, T.M. Guerra, R. Babuska, Perspectives of fuzzy systems and control, Fuzzy Sets Syst. 156 (3) (2005) $432-444$.

[6] E. Kim, H. Lee, New approaches to relaxed quadratic stability condition of fuzzy control systems, IEEE Trans. Fuzzy Sets 8 (5) (2000) 523-534.

[7] L. Xiaodiong, Z. Qingling, New approach to $H_{\infty}$ controller designs based on observers for T-S fuzzy systems via LMI, Automatica 39 (2003) 1571-1582.

[8] M. Chadli, A. Akhenak, J. Ragot, D. Maquin, State and unknown input estimation for discrete time multiple model, J. Frankl. Inst. 346 (6) (2009) 593-610.

[9] M. Chadli, S. Aouaouda, H.R. Karimi, P. Shi, Robust fault tolerant tracking controller design for a VTOL aircraft, J. Frankl. Inst. 350 (9) (2013) 2627-2645.

[10] T. Youssef, M. Chadli, H.R. Karimi, M. Zelmat. Design of unknown inputs proportional integral observers for TS fuzzy models. Neurocomputing 123(10) (2014), 156-165.

[11] M. Johansson, A. Rantzer, K. Arzén, Piecewise quadratic stability of fuzzy systems, IEEE Trans. Fuzzy Syst. 7 (6) (1999) 713-722.

[12] M. Chadli, H.R. Karimi, Robust observer design for unknown inputs Takagi-Sugeno models, IEEE Trans. Fuzzy Syst. 12(1) (2013) 158-164.

[13] K. Tanaka, T. Hori, H.O. Wang, A multiple Lyapunov function approach to stabilization of fuzzy control systems, IEEE Trans. Fuzzy Syst. 11 (4) (2003) 582-589.

[14] L. Dai, Singular Control Systems, Lecture Notes in Control and Information Sciences, vol. 118, Springer-Verlag, New York, 1989.

[15] F.L. Lewis, A survey of linear singular systems, Circuits Syst. Signal Process. 5 (1986) 3-36.

[16] R.W. Newcomb, The semistate description of nonlinear time-variable circuits, IEEE Trans. Circuits Syst. 28 (1981) $62-71$.

[17] S. Xu, J. Lam, Robust Control and Filtering of Singular Systems, Springer, Berlin, Heidelberg, New York, 2006 ISBN-10 3-540-32797-5.

[18] E. Fridman, A Lyapunov-based approach to stability of descriptor systems with delay, in: Proceedings of the 40th IEEE Conference on Control and Decision, Orlando, FL, USA, December 2001, pp. 2850-2855.

[19] W. Lam, J. Huang, Semiglobal stabilization and output regulation of singular linear systems with input saturation, IEEE Trans. Autom. Control 48 (7) (2003) 1274-1280.

[20] S. Xu, P.V. Dooren, R. Stefan, J. Lam, Robust stability and stabilization of discrete-time singular systems with state delay and parameter uncertainty, IEEE Trans. Autom. Control 47 (7) (2002) 1122-1128. 
[21] S. Xu, J. Lam, Robust stability and stabilization of discrete-time singular systems: an equivalent characterization, IEEE Trans. Autom. Control 49 (4) (2004) 568-574.

[22] S. Xu, C. Yang, Stabilization of discrete-time singular systems: a matrix inequalities approach, Automatica 35 (1999) 1613-1617.

[23] L.R. Fletcher, Pole assignment and controllability subspaces in descriptor systems, Int. J. Control 66 (1997) 677-709.

[24] G.C. Verghese, B.C. Levy, T. Kailath, A generalized state-space for singular systems, IEEE Trans. Autom. Control 26 (1981) 811-831.

[25] S. Xu, J. Lam, L. Zhang, Robust D-stability analysis for uncertain discrete singular systems with state delay, IEEE Trans. Circuits Syst. I 49 (2002) 551-555.

[26] K. Takaba, N. Morihira, T. Katayama, A generalized Lyapunov theorem for descriptor system, Syst. Control Lett. 24 (1995) 49-51.

[27] S. Xu, J. Lam, Robust stability for uncertain discrete singular systems with state delay, Asian J. Control 5 (2003) 399-405.

[28] P. Shi, E.K. Boukas, On $H_{\infty}$ control design for singular continuous-time delay systems with parametric uncertainties, Nonlinear Dyn. Syst. Theory 4 (1) (2004) 59-71.

[29] C.-P. Huang, Stability analysis of discrete singular fuzzy systems, Fuzzy Sets Syst. 151 (2005) $155-1655$.

[30] S. Xu, B. Song, J. Lu, J. Lam, Robust stability of uncertain discrete-time singular fuzzy systems, Fuzzy Sets Syst. 158 (20) (2007) 2306-2316.

[31] C.H. Lee, J.-H. Wang, Y.-J. Chiu, S.-Y. Hsu, Control of singular fuzzy systems with time delays, in: SICE Annual Conference, 2010.

[32] C.H. Wang, T.M. Huang, J.B. Qiu, Robust $H_{\infty}$ control of discrete-time singular T-S fuzzy systems, in: Proceedings of the 7th World Congress on Intelligent Control and Automation, 2008.

[33] L. Wu, X. Su, P. Shi, Sliding mode control with bounded $L_{2}$ gain performance of Markovian jump singular timedelay systems, Automatica 48 (8) (2012) 1929-1933.

[34] L. Wu, P. Shi, H. Gao, State estimation and sliding mode control of Markovian jump singular systems, IEEE Trans. Autom. Control 55 (5) (2010) 1213-1219.

[35] M. Chadli, A. Abdo, S.X. Ding, $H_{-}$and $H_{\infty}$ fault detection filter design for discrete-time Takagi-Sugeno fuzzy system, Automatica 49 (7) (2013) 1996-2005.

[36] M. Chadli, M. Darouach, Robust admissibility of uncertain switched singular systems, Int. J. Control 84 (10) (2011) 1587-1600.

[37] P.-L. Liu, Improved delay-dependent robust exponential stabilization criteria for uncertain time-varying delay singular systems, Int. J. Innov. Comput. Inf. Control 9 (1) (2013) 165-178.

[38] P.-L. Liu, Further results on the exponential stability criteria for time delay singular systems with delay-dependence, Int. J. Innov. Comput. Inf. Control 8 (6) (2012) 4015-4024.

[39] F. Li, X. Zhang, Delay-range-dependent robust $H_{\infty}$ filtering for singular LPV systems with time variant delay, Int. J. Innov. Comput. Inf. Control 9 (1) (2013) 339-353.

[40] M. Chadli, M. Darouach, Novel bounded real lemma for discrete-time descriptor systems. Application to $H_{\infty}$ control design, Automatica 48 (2) (2012) 449-453.

[41] G. Zhang, Y. Xia, P. Shi, New bounded real lemma for discrete-time singular systems, Automatica 44 (3) (2008) 886-890.

[42] M. Chadli, M. Darouach, Further enhancement on robust $H_{\infty}$ control design for discrete-time singular systems, IEEE Trans. Autom. Control. doi: http://dx.doi.org/10.1109/TAC.2013.2273266, 2013.

[43] J. Löfberg, YALMIP: Software for solving convex (and nonconvex) optimization problems, in: American Control Conference, June 14-16, Minneapolis, MN, 2006. 